\title{
Challenges of cannabinoid delivery: how can nanomedicine help?
}

\author{
Emmanuel Shan Onaivi*,1,2 (iD), Bhanu Pratap Singh Chauhan²,3 \& Venkatanarayanan \\ Sharma ${ }^{1,2}$ \\ ${ }^{1}$ Department of Biology, College of Science \& Health, William Paterson University, Wayne, NJ 07470, USA \\ ${ }^{2}$ College of Science \& Health, William Paterson University, Wayne, NJ 07470, USA \\ ${ }^{3}$ Department of Chemistry, College of Science \& Health, William Paterson University, Wayne, NJ 07470, USA \\ *Author for correspondence: Onaivie@wpunj.edu
}

"There is an increasing global acceptance and interest in the use of cannabinoid formulations in medicine, as well as in recreational use of cannabis"

First draft submitted: 26 May 2020; Accepted for publication: 6 June 2020; Published online:

26 June 2020

Keywords: cannabinoids $\bullet$ cannabis $\bullet$ endocannabinoids $\bullet$ nanomedicine $\bullet$ nanoparticles $\bullet$ nanotherapeutics $\bullet$ theranostics

The cannabis plant contains a system that naturally produces molecules labeled as cannabinoids, terpenes and flavonoids which has ushered in a new era of medicine. There is an increasing global acceptance and interest in the use of cannabinoid formulations in medicine, as well as in recreational use of cannabis $[1,2]$. These molecules from the cannabis plant have the potential to treat numerous diseases. The rapidly shifting landscape and growing body of clinical evidence for the therapeutic efficacy of cannabinoids is due in part to new knowledge on cannabis molecular genetics, decoding its genome and the determination of CB1R and CB2R cannabinoid receptor crystal structures, along with their heteromeric complex formations. This paradigmatic progress has transformed cannabis research into mainstream science with remarkable advances, and the discovery of the human endocannabinoid system (ECS). The endocannabinoidome refers to the extended ECS including CB1, CB2, CB3 cannabinoid receptors and other candidates that are activated by cannabinoids, endocannabinoids and related compounds along with their metabolic enzymes [1]. In addition, the cellular, molecular, biochemical and behavioral responses to cannabinoids and cannabis use are encoded in our genome by CNR1 and CNR2 genes that have been mapped to human chromosomes 6 and 1, respectively [2].

This previously unknown but ubiquitous and expanded complex ECS is involved in the regulation of several functions in mammalian physiology and pathology, presenting potential targets that are being exploited for cannabinoid-based therapies. Scientists are now beginning to understand the complex pharmacology of cannabinoids and the other constituents found in cannabis, with a rapidly advancing innovative cannabinoid therapeutic options. Indeed, the conclusions of the US National Academies of Sciences, Engineering and Medicine provides the current state of evidence and recommendations for research on the health effects of cannabis and cannabinoids [3]. Such evidence-based scientific knowledge supports novel approaches for cannabinoid-based nanoformulation and cannabis use in theranostic nanomedicine. Therefore, progress in clinical development of nanomedicines due in part to advances in nanotechnology is fueling the development of multifunctional nanocarrier strategies as drug-delivery systems $[4,5]$. Nanopharmaceutical products using nanostrategies are already in clinical use and this commentary describes the ongoing nanotechnological developments that can overcome cannabinoid delivery challenges for optimal therapeutic effects.

\section{The concept of cannabis \& cannabinoid theranostic nanomedicines}

The concept of cannabinoid theranostics are challenging to develop but they have potential applications and advantages for a number of disease conditions, that are not adequately addressed with existing medicines. Although cannabis has been used as a medicine for millennia, in recent years there has been an increased interest in 
legalizing cannabis for medicinal use in AIDS, cancer, multiple sclerosis, post-traumatic-stress disorder (PTSD), nausea, anorexia, cachexia, neuropathic pain, drug dependency, neurological and other psychiatric disorders [1]. A nonintoxicating cannabinoid from the cannabis plant, cannabidiol (CBD), also called Epidiolex ${ }^{\circledR}$, has been approved by the US FDA for the treatment of epileptic seizures, Lennox-Gastaut and Dravet syndrome in children 2 years and older [1]. The medicinal and recreational use of cannabis is increasing as countries worldwide are setting up official programs to provide access to safe sources, and questions of formulations for safe delivery systems and regulations must be addressed for traditional and nanomedicine approaches. New knowledge affirming the traditional millennia use of cannabis for antiemetic, analgesic, anti-inflammatory and antiproliferative properties are also fueling this global trend and catalyzing the wave of cannabis programs. This multiplicity of cannabis products, pharmaceuticals and edibles are opening new opportunities and advances in routes of administration and delivery systems for cannabis products and medicines. As cannabinoids are a class of lipophilic compounds, the use of different surfactants and delivery systems to improve cannabinoid solubility and enhance bioavailability must be considered. The physicochemical properties associated with poor long-term stability, and psychoactive side effects present further challenges with cannabinoid delivery $[6,7]$.

Nanotechnological advances and increasing scientific knowledge on delivery systems and dosage requirements for specific therapeutic indications could be used to overcome these barriers to developing cannabis and cannabinoid theranostic nanomedicines. Indeed, with increasing opportunities and challenges in the formulation of cannabis products and quality control and safety issues, there is a growing research activity on cannabis and cannabinoids in nanomedicine. In practice, this means that the cannabinoids can be loaded into or conjugated to nanoparticles, offering the opportunity to effectively deliver the cannabis and cannabinoid formulations to target diseased sites. The application of nanotechnology in medicine involves employing nanoparticles not only to enhance the action of drugs, but also to improve the diagnosis and therapy of diseases and reduce healthcare costs [6]. The higher efficacy, reduction in toxicity, targeted release and control of biodistribution of the drug provides nanoparticle-based medicinal advantages over traditional medications. Many pharmaceutical products using strategies and devices incorporating nanovehicular drug carriers as nanoparticle-based medicines are already in clinical use [8]. Thus, nanotechnology may allow targeted delivery of cannabinoid formulations with the potential to elevate their use to scientifically validated nanotherapeutic and theranostic applications as the field of cannabis nanoscience matures.

\section{Nanoparticle cannabinoid conjugates}

Nanotechnological innovations has opened 'theranostic medicine', facilitating the development of combined diagnostic and therapeutic strategies with demonstrable potential in malignant gliomas [9]. Nanoconjugation and encapsulation strategies have been demonstrated to be a strategy for improving drug delivery and therapeutic efficacy of poorly water-soluble drugs [7]. Thus, cannabinoid nanoformulations using nanoconjugation strategies for biomedical applications can be considered in attempts to overcome drug delivery challenges to improve bioavailability, safety, diagnosis and efficacy. Specifically, cannabinoid theranostic nanoparticles have translational potential in breast cancer and in targeting components of the ECS in a number of health disorders [10,11].

Development and recent advances in nanocarrier-mediated delivery of bioactive molecules of plant origin is derived from the structure and properties of the nanocarrier and the controlled release of the phytocannabinoid [7,9]. The new frontiers of cannabis science, and the availability of the human genome and draft genome of the cannabis plant [12], are providing and allowing the examination of the development of therapeutic cannabis strains with tailored profiles or the bioengineering or genetic modification of cannabinoids in yeast for use as cannabinoid nanoconjugates [13]. There are over 100 cannabinoids in the cannabis plant, many of which have not yet been functionally characterized [14], and whether these bioengineering platforms present a cheaper way to produce natural and synthetic cannabinoids for use in nanoformulations for specific biomedical indications remains to be elucidated. However, investigation into this area could allow the development of multifunctional cannabinoid-nanocarriers with potential to treat a variety of diseases especially in conditions of endocannabinoid insufficiency.

\section{Cannabinoid nanodelivery systems}

In the last decade, the success of new and innovative nano synthetic tools has created novel theranostic opportunities in drug design that can no longer be considered futuristic. Nanotechnology-based drug-delivery strategies allow the creation of drugs, prodrugs or diagnostic materials which have been approved in many therapeutic fields [7]. To improve efficacy as well as reduce side effects, the active substances used in nanoformulations for diagnostic and therapeutic applications possess unique nanosized mechanical, physical, chemical and optical properties. These 
properties make nanoscale systems suitable for targeted delivery to specific cells and tissues throughout the human body. Recent applications of cell membrane-coated nanoparticles now provide biomimetic systems for efficient drug delivery, but have not been fully developed [7,15].

Cannabinoid nanodelivery systems have been applied in vitro and in vivo to serve as therapeutic strategies. A number of studies are building nanoparticles for nanodelivery of cannabis, cannabinoids and ECS components as nanotherapeutics [6,16-26]. They include strategies for creating cannabinoid delivery systems incorporating cannabinoid nanoconjugates in a multifunctional nanocarrier. Such nanostructured carriers are metallic; categorized as inorganic, organic or hybrid nanosytems loaded as different types of nanocarriers, based on specific requirements and the route of administration. Encapsulation strategies for cannabis products and edibles that can be considered as solid delivery systems will require greater physical stability, protection against oxidation and flavor masking using liposomes, micelles, polyplexes, polymersomes and silica nanoparticles as the industry matures and develops. However, the research in the nanoengineering area is still not sufficiently mature to achieve this, but it is conceivable that the surface of nano objects can be tailored to control the solubility of the drugs, ensuring effective circulation time and limiting the biodistribution. In addition, this strategy allows controlled drug release, which in turn reduces and diminishes immunogenicity. One of the promising strategies has been to conjugate the nano object's surface with microenvironment specific and/or receptor-specific biomacromolecules such as peptides, proteins and aptamers. A review of such studies provides a comprehensive picture of accomplishments and bottlenecks in this research area [7]. Some of these nanoconjugates have shown excellent in vitro preclinical and clinical efficacy as summarized by these selected examples of cannabinoid-based nanodelivery systems described below.

The successful development of synthetic cannabinoids in nanomicelles, nanoemulsions and nanostructured lipid carriers (NLCs) for potential therapeutic applications have been demonstrated [18,19,21]. In preclinical studies, there is an evidence indicating that nanoparticles and nanoemulsions consisting of hemp oil provide a promising alternative to current formulation technologies and are suitable for the oral delivery of drugs with bioavailability issues [22]. Further, a nanomicellar formulation of a synthetic cannabinoid reduced tumor growth and psychoactive side effects in a syngenic mouse model of triple-negative breast cancer [18]. CBD, a nonintoxicating component in cannabis, was incorporated in lipid nanocapsules (LNCs) and was effective for prolonged-release against a human glioblastoma cell line in vitro [19]. The blood-brain barrier (BBB) limits drug penetration into the brain leading to poor prognosis and therapeutic outcomes for neurological conditions. While brain drug delivery is a challenging route of administration, nanoparticle drug delivery systems are showing promise in overcoming this barrier [4,21]. In preclinical studies, nose-to-brain administration is showing a promising increase in drug concentration in the brain. NLCs, solid lipid nanoparticles and polymeric nanoparticle-delivery systems were compared with drug delivery to the brain, and cannabinoid-loaded NLCs were shown to be better, indicating the potential of cannabinoid nanoformulations in overcoming the limitations of poor solubility and absorption, lipophilicity and oral bioavailability of cannabis and cannabinoids [18-21]. Furthermore, LNCs have been widely studied as nanocarriers for the encapsulation of lipophilic compounds including cannabinoids. CBD enhanced the passage of LNCs across the BBB both in vitro and in vivo and highest brain-targeting ability was achieved with the smallestsized cannabinoid-decorated LNCs [21]. In addition, the formulation optimization and long-term physicochemical stability of mucoadhesive tetrahydrocannabinol-loaded NLCs seems feasible [23]. Therefore, nanostructured lipid carrier-based formulations are promising drug-delivery systems with modifications of nanoparticle strategies not only for cannabis and cannabinoids but also for other therapeutic agents.

In order to avoid the intestinal first-pass effect, solubility, absorption and high lipophilicity of cannabinoids, advanced pro nanolipospheres and self-nano emulsifying drug delivery systems containing natural absorption enhancers on the oral bioavailability of tetrahydrocannabinol (THC) and CBD have been used in a rat model [7,24]. Cannabinoid-loaded poly(lactic-co-glycolic acid) PLGA nanoparticles also have potential for theranostic applications, for example, CBD-loaded PLGA nanoparticles were effective at inhibiting tumor growth following intraperitoneal administration in a model of ovarian cancer [25]. In a proof of concept study, it was demonstrated that green synthesis of gold and silver nanoparticles from cannabis could be used to fight infections caused by microbial biofilms [16]. In human volunteers, formulations based on pro nano dispersion technology improved oral cannabinoid bioavailability [17]. Nanoparticle formulation of a poorly soluble cannabinoid receptor 1 antagonist also improved absorption in rat and human intestines [26]. LNCs can be used as carriers for lipophilic drugs to overcome their low aqueous solubility. For example, Aparicio-Blanco et al., were able perform in vitro screening of LNCs decorated and loaded with CBD as targeted prolonged-release carriers for glioma therapy [19]. LNCs have also been examined as carriers of ECS components and monoglyceride-LNCs have demonstrated potential for encapsu- 
lation and optimization of encapsulation of cannabinoid drugs [20]. Indeed, it has been reported that cannabinoid receptors are the most abundant G-protein coupled receptors (GPCRs) in the CNS and can be therapeutic targets for psychiatric and neurological disorders [27]. Application of cell membrane-based cannabinoid delivery systems may have advantages in providing biomimetic systems for efficient delivery of cannabinoid nanomedicines, but these have not yet been fully developed [15].

Our 'Nanomaterials Laboratory' group at William Paterson University targets developing new materials with unique optical, electrical and signaling properties, which are not available in traditional nanosystems [28]. We have been exploring various synthetic strategies to tailor metallic and semiconductor nanoparticles with various silicon-based biocompatible oligo- or polymers to manipulate the stability, solubility and delivery of drugs in a predictable fashion. This strategy can provide control over drug delivery in cases where traditional oral or injectable drug formulations cannot be used. We are also trying to generate new nanoparticle systems loaded with cytokines and cannabinoids for theranostic application in viral infections inducing inflammation [29]. The approach is to synthesize drug conjugates, which are inert, biocompatible, mechanically strong and safe. The goal is to incorporate cannabinoid nanoparticles in our mechanistic in vitro and in vivo studies taking into account particle size and route of delivery of the cannabinoid molecules in transgenic mice models of CNS function.

\section{Conclusion \& future perspective}

The growing acceptance of cannabis and cannabinoids raises questions and concerns not only about safety and therapeutic effects, but also about formulation and delivery strategies that bypass previous challenges. Nanotechnology is already transforming multiple facets of drug delivery in healthcare and the future of cannabis and cannabinoid use in nanomedicine offers promising solutions for many disease conditions. While the low aqueous solubility, low cell penetration and susceptibility to degradation of lipophilic cannabinoids present sustained delivery issues, progress in the formulation of targeted cannabinoid-nanocarrier delivery systems using different materials and techniques, provides unique opportunities to protect these compounds from degradation $[6,7,10,11]$. Many carrier systems have been studied, including liposomes, polymeric micelles, microspheres and nanoparticles and the choice of carrier depends on the molecular target for delivery. However, solid lipid nanoparticles show major advantages such as sustained release, improved bioavailability, improved drug incorporation and a wide range of applications [7,20]. CNS disorders are a key therapeutic targets for cannabis and cannabinoids, and nanoformulation platforms for cannabinoid nanoconjugates that provides efficient transportation across the BBB and limit opsonization are most desirable. The selected examples of cannabinoid nanoconjugates showed enhanced bioavailability and improved bio-efficacy with promising outcomes in biomedical applications.

The set back and withdrawal of the CB1R antagonist Acomplia as an antiobesity medication in Europe because of suicide inducing side effects in some patients, and the severe adverse effects seen during the clinical trial of the fatty acid amide hydrolase inhibitor [30] may benefit from nanomedical strategies. We believe that significant progress is being made, and that new generations of cannabinoid nanoparticle-based approaches will play an important role in human disease and healthcare and that future studies will allow the development of strategies to overcome the challenges that limit the use of cannabinoids.

\footnotetext{
Financial \& competing interests disclosure

ES Onaivi is partly supported by William Paterson Assigned Release Time and is a Guest Researcher at the NIDA-Intramural Research Program, a part of the National Institutes of Health. BPS Chauhan gratefully acknowledged the ART support from the College of Sciences and health. The authors have no other relevant affiliations or financial involvement with any organization or entity with a financial interest in or financial conflict with the subject matter or materials discussed in the manuscript apart from those disclosed. No writing assistance was utilized in the production of this manuscript.
}

Open access

This work is licensed under the Attribution-NonCommercial-NoDerivatives 4.0 Unported License. To view a copy of this license, visit http://creativecommons.org/licenses/by-nc-nd/4.0/ 


\section{References}

1. Cristino L, Bisogno T, Di Marzo V. Cannabinoids and the expanded endocannabinoid system in neurological disorders. Nat. Rev. Neuro. 16(1), 9-29 (2020).

2. Joshi N, Onaivi ES. Endocannabinoid system components: overview and tissue distribution. In: Recent Advances in Cannabinoid Physiology and Pathology Bukiya (Ed.). Adv. Exp. Med. Biol. Springer Nature, Cham, Switzerland, 1162, 1-12 (2019).

3. National Academies of Sciences, Engineering, and Medicine. In: The Health Effects of Cannabis and Cannabinoids: The Current State of Evidence and Recommendations for Research. The National Academies Press, DC, USA (2017).

4. Mulvihill JJE, Cunnane EM, Ross AM, Duskey JT, Tosi G, Grabrucker AM. Drug delivery across the blood-brain barrier: recent advances in the use of nanocarriers. Nanomedicine (Lond.) 15(2), 205-214 (2020).

5. Singh D, Dilnawaz F, Sahoo SK. Challenges of moving theranostic nanomedicine into the clinic. Nanomedicine (Lond.) 15(2), 111-114 (2020).

6. Ngwa W, Kumar R, Moreau M, Dabney R, Herman A. Nanoparticle to target lung cancer with radiosensitizers and cannabinoids. Front. Oncol. 7, 208 (2017).

7. Bruni N, Pepa CD, Oliaro-Bosso S, Pessione E, Gastaldi D, Dosio F. Cannabinoid delivery systems for pain and inflammation treatment. Molecules 23, 2478 (2018).

8. Bobo D, Robinson KJ, Islam J, Thurecht KJ, Corrie SR. Nanoparticle-based medicines: a review of FDA-approved materials and clinical trials to date. Pharm. Res. 33, 2373-2387 (2016).

9. Wang L, Mori W, Cheng R. Synthesis and preclinical evaluation of sulfonamide-based $\left[{ }^{11} \mathrm{C}\right.$-Carbonyl]-carbamates and ureas for imaging monoacylglyerol lipase. Theranostics 6(8), 1145-1159 (2016).

10. Grimsey NL, Savinaine JR, Attili Ahmed M. Regulating membrane lipid levels at the synapse by small-molecule inhibitors of monoacylglycerol lipase: new developments in therapeutic and PET imaging applications. Drug Discov. Today 25(2), 330-343 (2020).

11. Mokoena DR, George BP, Abrahamse H. Enhancing breast cancer treatment using a combination of cannabidiol and gold nanoparticles for photodynamic therapy. Int. J. Mol. Sci. 20, 4771 (2019).

12. Gao S, Wang B, Xie S et al. A high-quality reference genome of wild Cannabis sativa. Hortic. Res. 7, 73 (2020).

13. Luo X, Reiter MA, Espaux L et al. Complete biosynthesis of cannabinoids and their unnatural analogs in yeast. Nature 567, 123 (2019).

14. Leghissa A, Hildenbrand ZL, Schug KA. The imperatives and challenges of analyzing Cannabis edibles. Curr. Opin. Food Sci. 28, 18-24 (2019).

15. Varnamkhasti BS, Jafari S, Taghavi F et al. Cell penetrating peptides: as a promising theranostic strategy to circumvent the blood-brain barrier for CNS disease. Curr. Drug Deliv. 17, doi:10.2174/1577201817666200415111755 (2020).

16. Singh P, Pandit S, Garnaes J et al. Green synthesis of gold and silver nanoparticles from cannabis sativa (industrial hemp) and their capacity for biofilm inhibition. Int. J. Nanomedicine 13, 3571-3591 (2018).

17. Atsmon J, Cherniakov I, Izgelov D et al. PTL401, a new formulation based on pro-nano dispersion technology, improves oral cannabinoids bioavailability in healthy volunteers. J. Pharm. Sci. 107(5), 1423-1429 (2018).

18. Greish K, Mathur A, Al Zahrani R et al. Synthetic cannabinoids nano-micelles for management of triple-negative breast cancer. $J$. Control. Rel. 291, 184-195 (2018).

19. Aparicio-Blanco J, Sebastian V, Benoit JP, Torres-Suarez AI. Lipid nanocapsules decorated and loaded with cannabidiol as targeted prolonged-release carriers for glioma therapy: in vitro screening of critical parameters. Eur. J. Pharm. Biopharm. 134, 126-137 (2019)

20. Esposito E, Drechsler M, Cortesi R, Nastruzzi C. Encapsulation of cannabinoid drugs in nanostructured lipid carriers. Eur. J. Pharm. Biopharm. 102, 87-91 (2016).

21. Aparicio-Blanco J, Romero IA, Male DK, Slowing K, Torres-Suarez AI. Cannabidiol enhances the passage of lipid nanocapsules across the blood-brain barrier both in vitro and in vivo. Mol. Pharm. 16(5), 1999-2010 (2019).

22. Yin J, Xiang C, Wang P, Yin Y, Hou Y. Biocompatible nanoemulsions based on hemp oil and less surfactants for oral delivery of baicalein with enhanced bioavailability. Int. J. Nanomedicine 12, 2923-2931 (2017).

23. Hommoss G, Pyo SM, Muller RH. Mucoadhesive tetrahydrocannabinoil-loaded NLC - formulation optimization and long-term physicochemical stability. Eur. J. Pharm. Biopharm. 117, 408-417 (2017).

24. Cherniakov I, Izgelov D, Domb AJ, Hoffman A. The effect of pro nanolipospheres (PNL) formulation containing natural absorption enhancers on the oral bioavailability of delta-9-tetrahydrocannabinol (THC) and cannabidiol (CBD) in a rat model. Eur. J. Pharm. Sci. 109, 21-30 (2017).

25. Fraguas-Sanchez AI, Torres-Suarez AI, Cohen M et al. PLGA nanoparticles for intraperitoneal administration of CBD in the treatment of ovarian cancer: in vitro and in ovo assessment. Pharmaceutics 12(5), E439 (2020).

26. Siissalo $\mathrm{S}$, de Waard $\mathrm{H}$, de Jager $\mathrm{MH}$ et al. Nanoparticle formulation of a poorly soluble cannabinoid receptor 1 antagonist improves absorption by rat and human intestine. Drug Metab. Dispos. 41(8), 1557-1565 (2013).

27. Chiarlone A, Bellocchio L, Blazquez C et al. A restricted population of $\mathrm{CB} 1$ cannabinoid receptors with neuroprotective activity. Proc. Natl Acad. Sci. USA 111(22), 8257-8262 (2014). 
28. Chauhan BPS, Matam S, Johnson QR, Partel A, Moran K, Onyechi B. Generation of zerovalent metal core nanoparticles using n-(2-aminoethyl)-3-aminosilanetriol. J. Vis. Exp. 108, e53507 (2016).

29. Sharma V. Current perspectives on cytokines for anti-retroviral therapy in AIDS related B-cell lymphomas. Curr. Drug Targets Infect. Disord. 3, 137-149 (2003).

30. Hayes AW. Commentary on BIA 10-2474. Reg. Tox. Pharmacol. 111, 104541 (2020). 